\title{
Cell fibers promote proliferation of co-cultured cells on a dish
}

\begin{abstract}
Ai Shima ${ }^{1}$, Akane Itou ${ }^{2} \&$ Shoji Takeuchi ${ }^{1,2,3^{*}}$
This paper describes a co-culture method using cell fiber technology. Cell fibers are cell-laden hydrogel microfibers, in which cells are cultured three-dimensionally and allowed to reach more mature state than the conventional two-dimensional cell culture. Cells in the cell fibers are encapsulated by alginate shell. Only cellular secretome is released into the surrounding environment through the shell while the cells were retained by the fiber. With their high handleability and retrievability, we propose to use the cell fibers for co-culture to ensure steady supply of cellular secretome. We cultured mouse $\mathrm{C} 2 \mathrm{C} 12$ myoblasts with mouse 3T3 fibroblasts encapsulated in the cell fibers for two days. The number of $\mathrm{C} 2 \mathrm{C} 12$ cells increased proportionally to the number of co-cultured 3T3 fibers, suggesting that the secretome of $3 \mathrm{~T} 3$ fibers promoted survival and proliferation of $\mathrm{C} 2 \mathrm{C} 12$ cells. We believe that cell fiber technology is a useful tool for co-culturing cells, and it will contribute to both basic cell biology and tissue engineering with its unique features.
\end{abstract}

Co-culture, in which two or more types of cells are cultured together, is a major method to study interactions between different types of cells in vitro. Cells interact with each other both directly (via physical contact) and indirectly (via secreted molecules; for example, cytokines, growth factors and hormones) and these interactions have an impact on cellular survival, proliferation, differentiation and maturation. To investigate the indirect cellular interactions, two major methods have been established; one using culture inserts and the other using conditioned medium. Culture inserts make upper and lower compartments in culture wells, which enables a concurrent co-culture. Two different types of cells are plated and cultured in the upper and lower compartments. Only cellular secretome, but not the cells themselves, is then transferred between those two compartments through the pores on the bottom of culture inserts, when the pore size is smaller than the cells. On the other hand, in the method that employs conditioned medium, a certain type of cells are cultured and the supernatant containing their secretome (conditioned medium) is collected to subsequently culture the other type of cells. These methods are often employed and have been shown to be effective to study various cellular interactions ${ }^{1-3}$. However, neither of them is highly space-efficient; the number of available cells that provides secretome is limited because of the culture area.

Cell fiber is a unique tool for culturing cells three-dimensionally for a long period until they differentiate into a mature tissue ${ }^{4}$. Cell fibers, which are cell-laden hydrogel microfibers formed by using a double-coaxial laminar-flow microfluidic device, consist of two parts; the core containing cells and extra cellular matrix (ECM) proteins such as collagen, and the alginate shell. Various types of cells have been shown to form three-dimensional (3D) tissues in cell fibers; for example, cardiomyocytes, vascular endothelial cells, nerve cells, smooth muscle cells and adipocytes ${ }^{4-6}$. Cell fibers enable a large number of cells to be packed together. The cells can easily access oxygen and nutrients in the culture medium, since thickness of the core containing the cells is kept a few hundreds of micrometers over the entire length. Also, they are mass-producible; by determining the flow rate and injection time of the core, cell fibers containing roughly the same number of cells can be repeatedly formed. Cell fibers are not only a useful tool for basic cell biology to study cellular behavior in a 3D culture, but also have the potential to be applied as grafts for cell therapy. It was demonstrated that the transplanted insulin-secreting cell fiber decreased blood glucose level in diabetic mice ${ }^{4,7}$. The advantage of cell fibers is that the alginate shell isolates the transplanted cells in the core from host immune system, which otherwise induces foreign-body reaction. The other advantage is their handleability (easily transplantable and retrievable).

In this study, we propose to use the cell fibers, which provide cell-derived components, for co-culture of different types of cells (the conceptual illustration of this study is shown in Fig. 1). Cell fibers contain a large

${ }^{1}$ Department of Mechano-Informatics, Graduate School of Information Science and Technology, The University of Tokyo, Tokyo, Japan. ${ }^{2}$ Institute of Industrial Science, The University of Tokyo, Tokyo, Japan. ${ }^{3}$ International Research Center for Neurointelligence (WPI-IRCN), The University of Tokyo Institutes for Advanced Study (UTIAS), The University of Tokyo, Tokyo, Japan. *email: takeuchi@hybrid.t.u-tokyo.ac.jp 


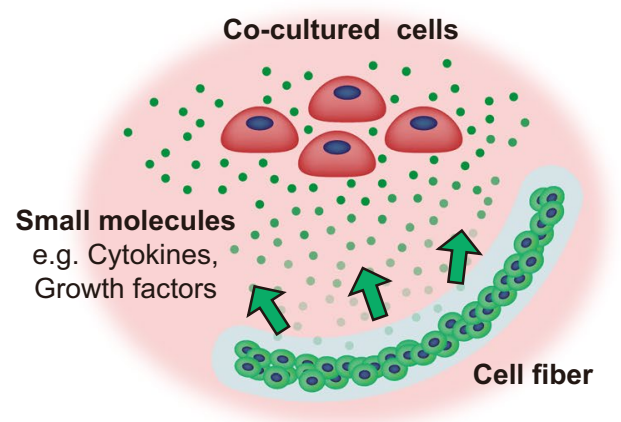

$\checkmark$ Secretome of a large number of cells $\checkmark$ No cell contamination

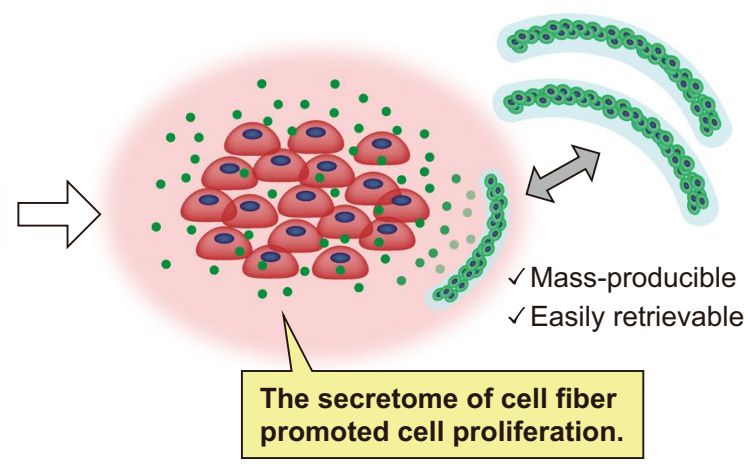

The secretome of cell fiber

Figure 1. Conceptual illustration of this study. A large number of cells in the cell fiber secrete small molecules such as cytokines and growth factors into the culture medium through the hydrogel shell, while the cells are retained by the fiber. Since cell fibers are mass-producible and can be easily retrieved from the culture, they are a useful tool to supply cell-derived components to other cells in co-culture. This study investigated whether the secretome of cell fibers promoted proliferation of co-cultured cells.

number of cells in less volume and are mass-producible. They can also be easily retrieved after the co-culture. Here, we culture mouse 3T3 fibroblasts encapsulated in the cell fibers and mouse C2C12 myoblasts together to investigate whether the secretome of $3 \mathrm{~T} 3$ fibers promotes the proliferation of $\mathrm{C} 2 \mathrm{C} 12$ cells without causing cellular contamination.

\section{Results}

Co-culture of $\mathrm{C} 2 \mathrm{C} 12$ cells and a $3 \mathrm{~T} 3$ fiber without cellular contamination. First, we monitored the formation of 3T3 fibers and their handleability for co-culture. After three days of culture, 3T3 cells adhered to each other and formed fiber-based 3D tissues in the alginate shell of the cell fibers (Fig. 2a,b). The diameter of alginate shell of 3T3 fibers was about $220 \mu \mathrm{m}$, whereas the diameter of the core (cells and ECM) was about $70 \mu \mathrm{m}$ on day 3 . A certain length of both ends of the $3 \mathrm{~T} 3$ fibers was composed of only alginate shell without the core containing cells (Fig. 2a), so that the cells would not leak out from the edges. Before starting co-culture with $\mathrm{C} 2 \mathrm{C} 12$ cells plated on tissue culture dishes, the $3 \mathrm{~T} 3$ fibers were observed carefully and confirmed to be intact. The entire length of cell fibers could be observed at low magnetic field by microscope. In case 3T3 fibers were found to have ruptures on the shell leading to leakage of the cells, they were not used for co-culture. The 3T3 fibers were cultured in Dulbecco's modified Eagle medium (DMEM) containing 10\% fetal bovine serum (FBS) after being formed. To avoid traces of FBS during transfer of the cell fibers into the serum-free C2C12 culture, the 3T3 fibers were washed in fresh DMEM (containing only antibiotics) for three times before being transferred. The cell fibers were then transferred to the dishes on which $\mathrm{C} 2 \mathrm{C} 12$ cells were cultured to investigate the effect of cellular secretome of $3 \mathrm{~T} 3$ fibers on $\mathrm{C} 2 \mathrm{C} 12$ cell proliferation (Fig. 2c). No visible damage was observed after the cell fibers were washed and transferred by using pipets. During the co-culture for two days, 3T3 cells in the cell fibers did not increase substantially and no cellular leakage was observed in the $\mathrm{C} 2 \mathrm{C} 12$ culture. These results suggested that 3T3 fibers were suitable for co-culture in terms of the handleability and sealing property.

The effect of a 3T3 fiber on $\mathrm{C} 2 \mathrm{C} 12$ cell proliferation. We then investigated whether the cellular secretome of a $3 \mathrm{~T} 3$ fiber could promote proliferation of $\mathrm{C} 2 \mathrm{C} 12$ cells in the co-culture. As a control to check the effects of absorbed FBS in the ECM core and alginate shell, which was possibly left even after the wash, an ECM-only fiber was employed (Fig. 2d). Just before a 3T3 fiber was transferred (day 0), the number of C2C12 cells per dish was $2.1 \times 10^{4}$ cells. C2C12 cells were then cultured with a 3T3 fiber or an ECM-only fiber in DMEM containing only antibiotics (serum-free medium), in the serum-free medium and in the C2C12 medium containing $5 \%$ FBS for two days (Fig. 3a). On day 2, the number of C2C12 cells cultured with a $3 \mathrm{~T} 3 \mathrm{fiber}\left(8.1 \times 10^{4}\right.$ cells $)$ was significantly higher $(P=0.013)$ than that cultured with an ECM-only fiber $\left(2.7 \times 10^{4}\right.$ cells $)$, whereas the difference between them was not statistically significant $(P=0.184)$ on day 1 . The $\mathrm{C} 2 \mathrm{C} 12$ cells cultured with an ECM-only fiber had hardly increased in two days. In the serum-free medium, the cell number even decreased, suggesting that the cells not only stopped proliferating but were also induced to undergo cell death. The co-culture with a $3 \mathrm{~T} 3$ fiber promoted $\mathrm{C} 2 \mathrm{C} 12$ cell proliferation, but the effect was not as high as the culture with 5\% FBS $\left(15.7 \times 10^{4}\right.$ cells on day 2) (Fig. 3b).

The expression of cell-proliferation marker $\mathrm{Ki}^{2} 7^{8}$ was also analyzed under above-mentioned conditions by immunofluorescence (Fig. 3c). The percentage of Ki67-positive C2C12 cells was highest when cultured in the $\mathrm{C} 2 \mathrm{C} 12$ medium containing 5\% FBS, followed by the culture with a $3 \mathrm{~T} 3 \mathrm{fiber}$, the culture with an ECM-only fiber, and the culture in the serum-free medium, which was the similar pattern with the cell number (Fig. 3d).

We also cultured $\mathrm{C} 2 \mathrm{C} 12$ cells in the conditioned medium (CM) obtained from confluent $3 \mathrm{~T} 3$ cells plated on a 100 -mm diameter dish (about $5 \times 10^{6}$ cells) for comparison. To study the effect of cellular secretome on cell proliferation in the absence of serum, it was ensured that the CM did not contain FBS, unlike the conventional CM culture. On day 2 , the number of $\mathrm{C} 2 \mathrm{C} 12$ cells cultured in $3 \mathrm{~T} 3 \mathrm{CM}$ was found to be reduced $\left(1.5 \times 10^{4}\right.$ cells $)$. The reason why CM was not as effective as a $3 \mathrm{~T} 3$ fiber in promoting $\mathrm{C} 2 \mathrm{C} 12$ cell proliferation might be the number of 
a

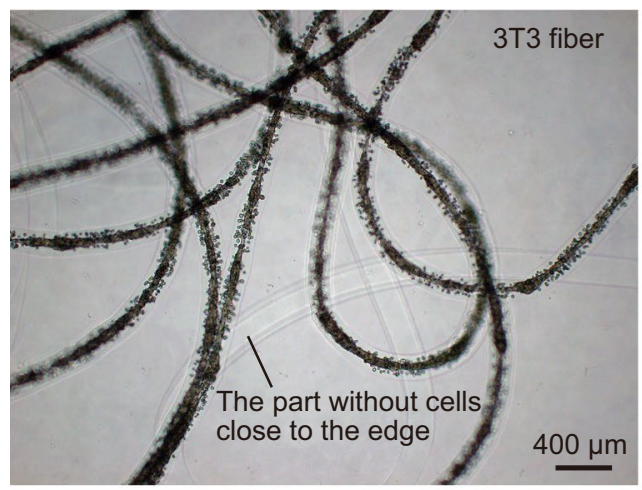

C

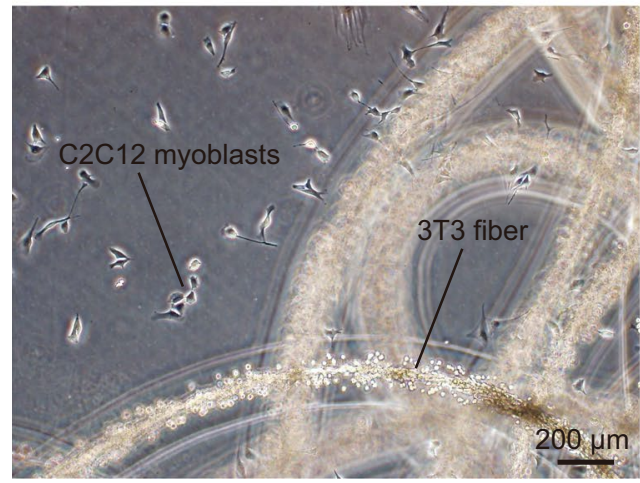

b

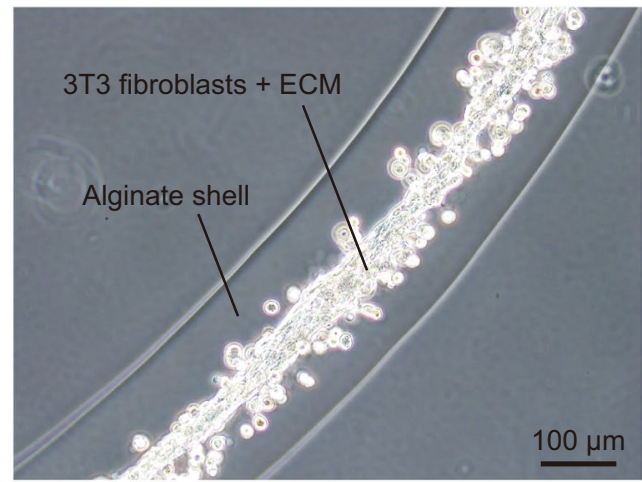

d

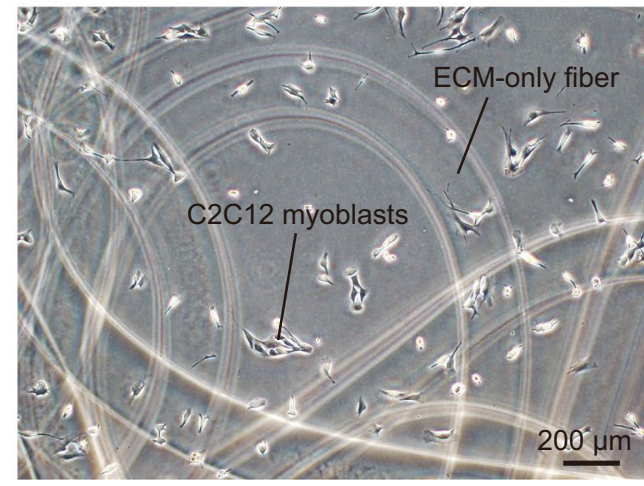

Figure 2. Co-culture of a 3T3 fiber and C2C12 cells. (a) Low-magnification and (b) high-magnification images of a 3T3 fiber three days after formation. The core consisting of 3T3 fibroblasts and ECM was sealed with the alginate shell. The part close to the edges did not contain the cells. (c) Co-culture of a 3T3 fiber and C2C12 cells. The day-3 3T3 fiber was transferred to C2C12 culture in a dish to initiate the co-culture. (d) Control culture of an ECM-only fiber and C2C12 cells.

3T3 cells cultured for CM $\left(10^{6}\right.$ order), which was much lower than that inside the cell fiber $\left(10^{7}\right.$ order $)$. In addition, the lack of serum may have deteriorated the culture condition of 3T3 cells when cultured in a dish without ECM, which also led to insufficient amounts of the secretome.

The effective factor in the secretome of a 3T3 fiber on $\mathrm{C} 2 \mathrm{C} 12$ cell proliferation. Enzyme-linked immunosorbent assay (ELISA) detected fibroblast growth factor (FGF) $2(13.8 \pm 1.9 \mathrm{pg} / \mathrm{ml})$ in the conditioned medium of a 3T3 fiber (serum-free medium cultured with a 3T3 fiber for two days without C2C12 cells), suggesting that one of the factors promoting $\mathrm{C} 2 \mathrm{C} 12$ cell proliferation in the co-culture would be FGF2.

The effect of cell fiber number on $\mathrm{C} 2 \mathrm{C} 12$ cell proliferation. Finally, we investigated whether the effect of $3 \mathrm{~T} 3$ fibers on $\mathrm{C} 2 \mathrm{C} 12$ cell proliferation depended on the number of cell fibers. We cultured $\mathrm{C} 2 \mathrm{C} 12$ cells with one, two and three 3T3 fibers for two days (Fig. 4a). The total volume of culture medium per dish was kept identical regardless of the number of 3T3 fibers. It was observed that $\mathrm{C} 2 \mathrm{C} 12$ cells increased proportionally to the number of $3 \mathrm{~T} 3$ fibers until day 2 (Fig. 4b). The number of $\mathrm{C} 2 \mathrm{C} 12$ cells when cultured with three $3 \mathrm{~T} 3$ fibers $\left(13.5 \times 10^{4}\right.$ cells on day 2$)$ was almost close to the number of $\mathrm{C} 2 \mathrm{C} 12$ cells cultured with $5 \%$ FBS, suggesting that the effect of $3 \mathrm{~T} 3$ fibers on $\mathrm{C} 2 \mathrm{C} 12$ cell proliferation could match the effect of serum when multiple 3T3 fibers were co-cultured at the same time.

\section{Discussion}

The current study proposed a co-culture system using cell fibers, micro-fiber-shaped cellular constructs in which cells were sealed by alginate gel. To demonstrate this concept, we co-cultured mouse C2C12 myoblasts plated on culture dishes with mouse 3T3 fibroblasts encapsulated in the cell fibers. The alginate shell was durable enough to seal 3T3 cells inside during the co-culture for two days. The 3T3 fibers did not show cell leakage during the co-culture. The number of C2C12 cells did not increase when cultured with an ECM-only fiber but quadrupled with a $3 \mathrm{~T} 3$ fiber in two days even without FBS, which suggested that the cellular secretome of 3T3 fibers promoted $\mathrm{C} 2 \mathrm{C} 12$ survival and proliferation. The cell proliferation-promoting effect was greater when the cells were co-cultured with multiple $3 \mathrm{~T} 3$ fibers.

Cells secrete various types of cytokines and growth factors into the surrounding microenvironment and the secretome changes the phenotype of other cells via autocrine or paracrine signaling ${ }^{9-11}$. The cellular secretome has been used to culture other cells in co-culture system and shown to be effective. In the current study, it was also shown that co-culturing with 3T3 fibers promoted proliferation of $\mathrm{C} 2 \mathrm{C} 12$ cells. It has been well-known for decades that fibroblasts have a paracrine effect on myoblasts ${ }^{12}$. The effective substances in the fibroblast secretome 
a

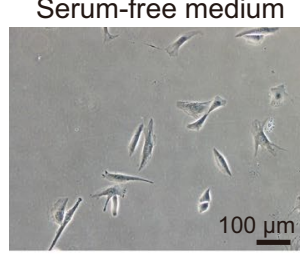

With 3T3 fiber

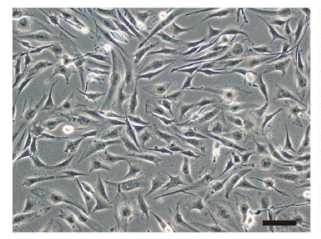

c
With ECM-only fiber

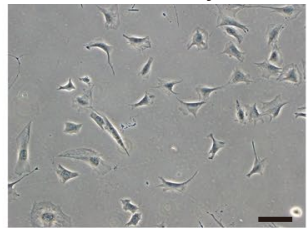

$5 \%$ FBS medium

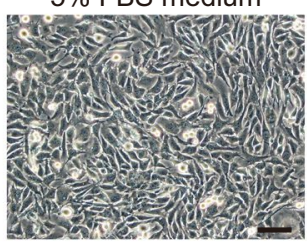

b

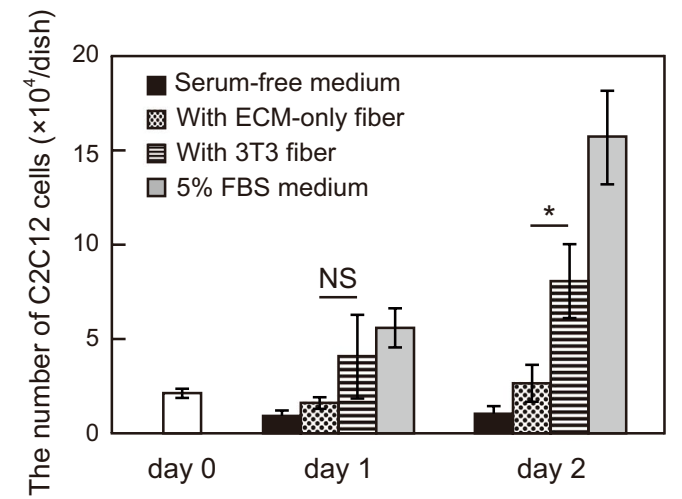

$5 \%$ FBS

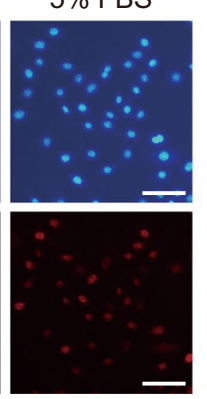

d

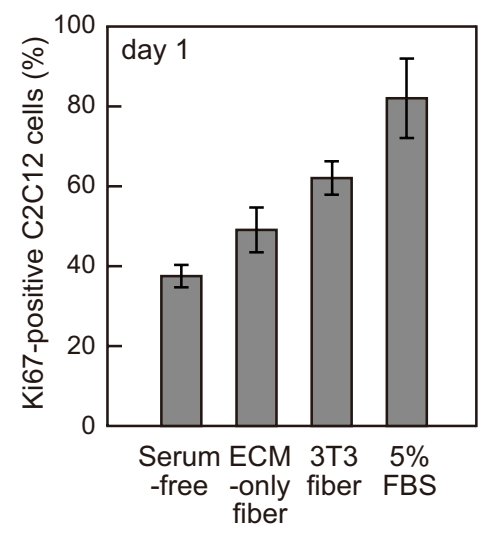

Figure 3. The effect of $3 \mathrm{~T} 3$ fibers on $\mathrm{C} 2 \mathrm{C} 12$ cell proliferation. (a) Images of $\mathrm{C} 2 \mathrm{C} 12$ cells after being cultured for two days in serum-free medium, with an ECM-only fiber, with a 3T3 fiber and in medium containing 5\% FBS. Scale bars; $100 \mu \mathrm{m}$. (b) The number of $\mathrm{C} 2 \mathrm{C} 12$ cells per culture dish under each condition on day 0 , day 1 and day 2. A 3 T3 fiber promoted C2C12 cell proliferation significantly more ( $* P=0.013$; $t$-test) than an ECM-only fiber on day 2 did. Data are mean \pm s.d. $(n=3)$. NS, not significant. (c) Images of immunofluorescence for the cell-proliferation marker, Ki-67 (red) under each condition on day 1. The cell nuclei were stained with Hoechst 33342 (blue). Scale bars; $100 \mu \mathrm{m}$. (d) The percentage of Ki67-positive C2C12 cells on day 1. The result showed a similar tendency in one of the cell number counting assays. Data are mean \pm s.d. $(n=4)$.

that stimulate myoblast proliferation were suggested to be growth factors such as FGF, insulin-like growth factor (IGF) and platelet-derived growth factor (PDGF) ${ }^{12}$. The current study also found that FGF2 was contained in the conditioned medium of a 3T3 fiber. The concentration of FGF2 was relatively low in the whole conditioned medium, but it would be higher in the immediate vicinity of 3T3 fibers in the co-culture. Recently, cellular secretome such as one from mesenchymal stem cells ${ }^{13-15}$ and myokine ${ }^{16-18}$ have been attracting attention in terms of their potential therapeutic effects, though some of them yet to be identified. It is difficult to determine the chemical composition of the secretome. However, we believe that cellular secretome is useful to promote cell growth in co-culture as some of the cell-derived components have not been artificially synthesized yet.

According to our results, the effect of $3 \mathrm{~T} 3$ fibers on $\mathrm{C} 2 \mathrm{C} 12$ cell proliferation was much greater than that of conditioned medium (CM) from $3 \mathrm{~T} 3$ cells. We assume that the main reason for this observation was the concentration of effective chemical factors in CM, which was about ten times lower than that of factors secreted in the co-culture with a $3 \mathrm{~T} 3$ fiber, since one tenth of $3 \mathrm{~T} 3$ cells encapsulated in a $3 \mathrm{~T} 3$ fiber were used to obtain the CM. In addition, 3T3 fibers could supply the secretome continuously and concurrently to C2C12 cells. C2C12 cells also could interact and change the behavior of 3T3 cells in the cell fibers, though no obvious effects on 3T3 fibers were observed in the current study probably because of the small number of plated $\mathrm{C} 2 \mathrm{C} 12$ cells. Note that CM used in this study did not contain FBS unlike the conventional CM culture, in which the cell-derived components are additionally incorporated into the general culture medium containing animal serum.

Animal serum such as FBS is essential for cell survival and proliferation in vitro. However, serum has its own drawbacks; for example, rising price, ethical concerns, possible pathogenicity, lot-to-lot variation and undefined animal-derived components ${ }^{19}$. We demonstrated that the cell-derived components from a commonly used cell line, 3T3, could become a substitute for serum when packed at sufficiently high cell concentration in a cell fiber and multiple cell fibers were applied. Cell lines are commercially available at a relatively lower price and pose no ethical issues. Due to their high proliferation ability, cell lines can be less expensive alternatives to animal serum when used in combination with the cell fiber technology. Not only general cell lines but also genetically engineered cells (overexpressing cells) or antibody-producing cells (hybridomas) can be encapsulated in cell fibers to oversecrete the intended chemical factors or to produce monoclonal antibodies, respectively. 

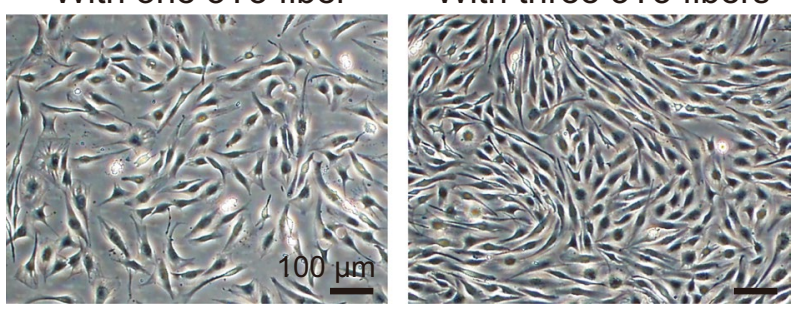

b

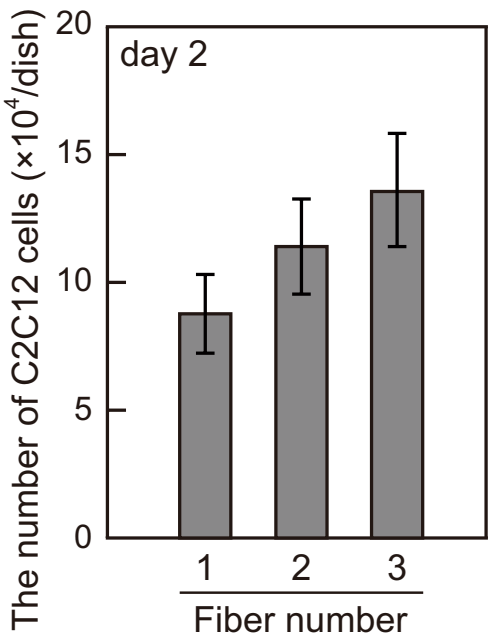

Figure 4. The effect of multiple $3 \mathrm{~T} 3$ fibers on $\mathrm{C} 2 \mathrm{C} 12$ cell proliferation. (a) Images of $\mathrm{C} 2 \mathrm{C} 12$ cells after being cultured for two days with one or three 3T3 fibers. Scale bars; $100 \mu \mathrm{m}$. (b) The number of C2C12 cells per dish under each condition on day 2. The increase in the number of $\mathrm{C} 2 \mathrm{C} 12$ cells was proportional to the number of 3 T3 fibers. Data are mean \pm s.d. $(n=4)$.

In conclusion, we demonstrated that 3T3 fibers promoted proliferation of co-cultured C2C12 cells. The cell fiber technology was useful to provide cellular secretome to the co-cultured cells. It is important in the research field of basic cell biology to elucidate various cellular interactions through paracrine signaling and study how the cells function in harmony in living organisms. We believe that the cell fiber technology will contribute to studies on cellular interactions with its unique features; scalability and handleability. Co-culture using cell fibers offers another advantage to the field of tissue engineering. Cell fibers can be co-cultured with the target cells not only in the standardized culture dishes but also in large tanks for industrial scale cell culture or original 3D culture devices, which have become more and more popular in recent years in the field of tissue engineering. Co-culture can be continued for more than two days by exchanging cell fibers regularly. Cell fibers can be retrieved after co-culture and the cultured tissues can be obtained without cellular contamination. We showed that the cell fibers can effectively provide secretome and can function as a less expensive alternative to animal serum. The compatibility of cell fibers with mass cell culture facilitate their contribution to the industrial field, for example cost-effective development of cultured meat ${ }^{19,20}$ in the future.

\section{Methods}

Cell culture. Mouse $\mathrm{C} 2 \mathrm{C} 12$ myoblasts ${ }^{21}$ were plated on tissue culture dishes coated with $1 \%$ gelatin (Sigma) at $1 \times 10^{4}$ cells per $35-\mathrm{mm}$ diameter dish and cultured in DMEM $(4500 \mathrm{mg} / \mathrm{mL}$ glucose) (Merck) containing $5 \%$ FBS (MP Biomedicals) and Penicillin-Streptomycin (Merck) (C2C12 medium) for $24 \mathrm{~h}$ before initiating co-culture with cell fibers or the control culture. For the cell fiber formation, mouse 3 T3 fibroblasts ${ }^{22}$ were expanded in DMEM (4500 mg/mL glucose) containing 10\% FBS and Penicillin-Streptomycin (3T3 medium). After the formation of 3T3 fibers and ECM-only fibers (mentioned below), both types of fibers were incubated in the 3T3 medium for three days. For the co-culture of $\mathrm{C} 2 \mathrm{C} 12$ cells and 3T3 fibers, the 3T3 fibers were confirmed to be intact (no leakage of cells from the core) by microscopic observation and washed with DMEM (without serum) for three times to get rid of FBS on the surface. One, two or three 3T3 fibers were then added into the culture of $\mathrm{C} 2 \mathrm{C} 12$ cells in $35-\mathrm{mm}$ diameter dishes with $2 \mathrm{~mL}$ of DMEM containing only antibiotics (serum-free medium). As a negative control, the ECM-only fibers were washed and cultured with $\mathrm{C} 2 \mathrm{C} 12$ cells. For other controls, the $\mathrm{C} 2 \mathrm{C} 12$ medium (containing 5\% FBS) and the serum-free medium were tested. For isolating conditioned medium (CM) from 3T3 cells, 3T3 cells were cultured in the 3T3 medium until they became confluent in 100-mm diameter dishes. The cells were then washed and incubated in the serum-free medium for $24 \mathrm{~h}$ and the supernatant was collected. The supernatant was centrifuged and filtered through a polyvinylidene difluoride (PVDF) membrane filter $(0.22 \mu \mathrm{m}$ pore $)$ to get rid of the cells and used as a CM to culture $\mathrm{C} 2 \mathrm{C} 12$ cells. All cultures were maintained in $5 \% \mathrm{CO}_{2}$ at $37^{\circ} \mathrm{C}$. The cell images were acquired with IX71 inverted research microscope (Olympus Life Science). 
Cell fiber formation. The cell fibers were formed by using the double-coaxial laminar-flow microfluidic device assembled from pulled glass capillary tubes, rectangular glass tubes and connectors as previously reported ${ }^{4}$. To form the core-shell $3 \mathrm{~T} 3$ fibers, three types of solutions were prepared: (1) 3T3 cells-containing pre-gel solution of a mixture of two types of collagen, AteloCell IAC-50 (Koken, Japan) and Cellmatrix Type I-A (Nitta Gelatin, Japan) as ECM for the core; (2) pre-gel solution of 1.5\% Na-alginate (Fujifilm Wako Pure Chemical, Japan) for the shell; (3) $100 \mathrm{mM} \mathrm{CaCl}_{2}, 3 \%$ glucose solution for the sheath stream. Each $3 \mathrm{~T} 3$ fiber was formed to contain $4 \times 10^{7}$ cells per fiber on the calculation with the certain length of both ends lacking cells (only alginate shell) to completely seal the cells. For the control fibers, only ECM without the cells was used to make the core (ECM-only fiber). The width of the shell and core parts of 3T3 fibers in randomly selected 18 fields from four different cell fiber formation trials were measured by using the imaging software cellSens (Olympus Life Science).

Cell counting. C2C12 cells were trypsinized after washing twice with PBS (the 3T3 fiber was taken out in such cases) and re-suspended in $1 \mathrm{~mL}$ of the $\mathrm{C} 2 \mathrm{C} 12$ medium, just before initiating co-culture with a $3 \mathrm{~T} 3$ fiber (day 0 ) and after being co-cultured for one or two days (day 1 and day 2, respectively). The cell number per dish was counted three times by using cell-counting plate (WakenBtech, Japan) and averaged. Under each experimental condition, three or four culture dishes from different cell culture trials were assayed. The number of cells per dish was shown as the mean \pm standard deviation (s.d.). The significance between the cell number with a 3T3 fiber and that with an ECM-only fiber was evaluated by $t$-test.

Immunofluorescence for Ki-67. C2C12 cells were fixed with 4\% paraformaldehyde/PBS after one-day co-culture with a $3 \mathrm{~T} 3$ fiber, when the cells under all experimental conditions were supposed to be in proliferating phase. The cells were permeabilized with $0.2 \%$ triton X-100/PBS, incubated with $1 \%$ BSA/PBS for blocking and treated with the primary antibody, rabbit polyclonal anti-Ki67 antibody (Abcam). Alexa Fluor 594 goat anti-rabbit IgG (Thermo Fisher Scientific) was used as the secondary antibody. Cell nuclei were stained with Hoechst 33342 (Thermo Fisher Scientific). The immunofluorescence images were taken with IX71 inverted research microscope. The percentages of Ki67-positive cells to the total cells were calculated by counting more than 300 cells in randomly selected six fields per dish in each condition. Four culture dishes from different cell culture trials were assayed and the results were shown as the mean \pm s.d.

ELISA for the detection of the secretome of a 3T3 fiber. Two mL each of DMEM containing only antibiotics (serum-free medium) cultured with a 3T3 fiber for two days without C2C12 cells (conditioned medium of a 3T3 fiber) from three culture dishes was analyzed. The conditioned medium was centrifuged at $400 \mathrm{~g}$ for $10 \mathrm{~min}$ at $4{ }^{\circ} \mathrm{C}$ and the supernatant was collected and stored at $-80^{\circ} \mathrm{C}$. ELISA was performed using Mouse/Rat FGF basic/FGF2 Quantikine ELISA Kit (R\&D Systems) according to the manufacturer's instructions. The signal was detected by using Cytation 5 Cell Imaging Multi-Mode Reader (BioTek Instruments). The concentration of FGF2 in the conditioned medium of a 3 T3 fiber was evaluated by a calibration curve and shown as the mean \pm s.d.

\section{Data availability}

The data that support the findings in this study are available from the corresponding author upon reasonable request.

Received: 22 August 2019; Accepted: 19 December 2019;

Published online: 14 January 2020

\section{References}

1. Seo, K., Suzuki, T., Kobayashi, K. \& Nishimura, T. Adipocytes suppress differentiation of muscle cells in a co-culture system. Anim. Sci. J. 90, 423-434 (2019).

2. Ostrovidov, S. et al. Three-dimensional co-culture of $\mathrm{C} 2 \mathrm{C} 12 / \mathrm{PC} 12$ cells improves skeletal muscle tissue formation and function. J. Tissue Eng. Regen. Med. 11, 582-595 (2017).

3. Shima, A. et al. IGF-I and vitamin C promote myogenic differentiation of mouse and human skeletal muscle cells at low temperatures. Exp. Cell Res. 317, 356-366 (2011).

4. Onoe, H. et al. Metre-long cell-laden microfibres exhibit tissue morphologies and functions. Nat. Mater. 12, 584-590 (2013).

5. Hsiao, A. Y. et al. Smooth muscle-like tissue constructs with circumferentially oriented cells formed by the cell fiber technology. PLoS One. 10, e0119010 (2015).

6. Hsiao, A. Y., Okitsu, T., Teramae, H. \& Takeuchi, S. 3D tissue formation of unilocular adipocytes in hydrogel microfibers. Adv. Healthc. Mater. 5, 548-556 (2016).

7. Ozawa, F., Okitsu, T. \& Takeuchi, S. Improvement in the mechanical properties of cell-laden hydrogel microfibers using interpenetrating polymer networks. ACS Biomater. Sci. Eng. 3, 392-398 (2017).

8. Sellathurai, J., Cheedipudi, S., Dhawan, J. \& Schrøder, H. D. A novel in vitro model for studying quiescence and activation of primary isolated human myoblasts. PLoS One. 8, e64067 (2013).

9. Skalnikova, H., Motlik, J., Gadher, S. J. \& Kovarova, H. Mapping of the secretome of primary isolates of mammalian cells, stem cells and derived cell lines. Proteomics. 11, 691-708 (2011).

10. Nicholson, T., Church, C., Baker, D. J. \& Jones, S. W. The role of adipokines in skeletal muscle inflammation and insulin sensitivity. J. Inflamm. 15, 9 (2018).

11. Bomb, R. et al. Myofibroblast secretome and its auto-/paracrine signaling. Expert. Rev. Cardiovasc. Ther. 14, 591-598 (2016).

12. Quinn, L. S., Ong, L. D. \& Roeder, R. A. Paracrine control of myoblast proliferation and differentiation by fibroblasts. Dev. Biol. 140, 8-19 (1990).

13. Praveen Kumar, L. et al. The mesenchymal stem cell secretome: a new paradigm towards cell-free therapeutic mode in regenerative medicine. Cytokine Growth Factor Rev. 46, 1-9 (2019).

14. Dietrich, J. et al. Analysis of lacrimal gland derived mesenchymal stem cell secretome and its impact on epithelial cell survival. Stem Cell Res. 38, 101477 (2019).

15. Harrell, C. R. et al. Molecular mechanisms responsible for therapeutic potential of mesenchymal stem cell-derived secretome. Cells. 8, 467 (2019). 
16. Whitham, M. \& Febbraio, M. A. The ever-expanding myokinome: discovery challenges and therapeutic implications. Nat. Rev. Drug Discov. 15, 719-729 (2016).

17. Son, J. S. et al. Effects of exercise-induced apelin levels on skeletal muscle and their capillarization in type 2 diabetic rats. Muscle Nerve. 56, 1155-1163 (2017).

18. Otaka, N. et al. Myonectin is an exercise-induced myokine that protects the heart from ischemia-reperfusion injury. Circ. Res. 123, 1326-1338 (2018).

19. Bhat, Z. F. \& Fayaz, H. Prospectus of cultured meat-advancing meat alternatives. J. Food Sci. Technol. 48, 125-140 (2011).

20. Post, M. J. Cultured meat from stem cells: challenges and prospects. Meat Sci. 92, 297-301 (2012).

21. Yaffe, D. \& Saxel, O. Serial passaging and differentiation of myogenic cells isolated from dystrophic mouse muscle. Nature. 270, 725-727 (1977).

22. Todaro, G. J. \& Green, H. Quantitative studies of the growth of mouse embryo cells in culture and their development into established lines. J. Cell Biol. 17, 299-313 (1963).

\section{Acknowledgements}

This work was supported by Grant-in-Aid for Scientific Research (S) (Grant number: 16H06329) and Grantin-Aid for Early-Career Scientists (Grant number: 18K18358) from Japan Society for the Promotion of Science (JSPS), and JST-Mirai Program (Grant Number JPMJMI18CE), Japan.

\section{Author contributions}

A.S. and S.T. designed the experiments and wrote the manuscript. A.S and A.I. performed the experiments. A.S. analyzed the data. All authors discussed the results and commented on the manuscript.

\section{Competing interests}

Shoji Takeuchi is an inventor on intellectual property rights related to the cell fiber technology, and stockholders of Cellfiber Inc., a start-up company based on the cell fiber technology.

\section{Additional information}

Correspondence and requests for materials should be addressed to S.T.

Reprints and permissions information is available at www.nature.com/reprints.

Publisher's note Springer Nature remains neutral with regard to jurisdictional claims in published maps and institutional affiliations.

Open Access This article is licensed under a Creative Commons Attribution 4.0 International License, which permits use, sharing, adaptation, distribution and reproduction in any medium or format, as long as you give appropriate credit to the original author(s) and the source, provide a link to the Creative Commons license, and indicate if changes were made. The images or other third party material in this article are included in the article's Creative Commons license, unless indicated otherwise in a credit line to the material. If material is not included in the article's Creative Commons license and your intended use is not permitted by statutory regulation or exceeds the permitted use, you will need to obtain permission directly from the copyright holder. To view a copy of this license, visit http://creativecommons.org/licenses/by/4.0/.

(C) The Author(s) 2020 